\title{
Coordination in the Decentralized Assembly System with Dual Supply Modes
}

\author{
Xu Guan ${ }^{1}$ and Mengqi Liu' ${ }^{2}$ \\ ${ }^{1}$ Economic and Management School, Wuhan University, Wuhan 430000, China \\ ${ }^{2}$ School of Business Administration, Hunan University, Changsha 410082, China \\ Correspondence should be addressed to Mengqi Liu; liumengqi1976@163.com
}

Received 27 June 2013; Revised 11 September 2013; Accepted 28 September 2013

Academic Editor: Tinggui Chen

Copyright (c) 2013 X. Guan and M. Liu. This is an open access article distributed under the Creative Commons Attribution License, which permits unrestricted use, distribution, and reproduction in any medium, provided the original work is properly cited.

\begin{abstract}
This paper investigates a decentralized assembly system that consists of one assembler and two independent suppliers; wherein one supplier is perfectly reliable for the production, while the other generates yield uncertainty. Facing the random market demand, the assembler has to order the components from one supplier in advance and meanwhile requires the other supplier to deliver the components under VMI mode. We construct a Nash game between the supplier and the assembler so as to derive their equilibrium procurement/production strategies. The results show that the channel's performance is highly undermined by the decentralization between players and also the combination of two supply modes. Compared to the centralized system, we propose an advance payment contract to perfectly coordinate the supply chain performance. The numerical examples indicate some management implications on the supply mode comparison and sensitivity analysis.
\end{abstract}

\section{Introduction}

Assembly systems have been widely applied in automobile, electronics, and many other manufacturing industries. One fundamental advantage of this system is to help the core assembler/manufacturer take the full advantages (e.g., low procurement cost) from his suppliers. Therefore, we have seen that many famous manufacturers (e.g., Toyota and HP) prefer to outsource their components production process to those external suppliers who locate in Asia, so as to significantly reduce their procurement cost and improve the production efficiency. Nonetheless, the accompanying challenge is how to ensure that the independent suppliers move cooperatively in the system. Difficulties arise from two aspects. First, because a single firm normally cares for its own profit, its decisions inevitably go against the channel's overall efficiency. Second, there are multiple uncertain factors that lie in the system, for example, delivery time, customer demand, and production yield, which subsequently undermine the system's operational performance. Therefore, to mitigate the decentralization and improve the system's performance, scholars have made extensive researches on the optimization mechanism in the decentralized assembly systems [1-4].
Nonetheless, in this rich stream of literature most scholars assume that the suppliers have to adopt the same strategies, in which all of them are either enrolled in the ordering mode or in the VMI mode (vendor managed inventory). Ordering mode is defined as were the assembler orders the components from all his suppliers before the demand is realized. As a result, suppliers just need to follow the assembler's instructions. For example, Yano [1] studies an assembler's optimal order time, wherein both suppliers have stochastic supply lead times. In contrast, under VMI mode all the suppliers have to personally determine when and how to produce the components to the assembler after knowing the demand information. For this issue, Gerchak and Wang [5] investigate how to achieve the coordination with both revenue-sharing contract and buy-back contract under VMI mode.

Differently, in this paper we will combine these two supply modes into a single assembly system, wherein one supplier exerts the ordering mode, while the other supplier has to accept the VMI mode. To our knowledge, this combination is rarely discussed in the prior literature but is prevalent in practice. For example, in China's auto industry, the assembler needs to order and pay for the key components, such as engine, from the overseas suppliers in advance. Meanwhile, 
the of the rest components such as windshield wipers are normally provided by the domestic suppliers under VMI mode. Also in the electronics industry, assembler always places orders in advance from key components' provider (e.g., Intel) while requires the local suppliers to produce the other components under VMI arrangement. Given this gap between the practice and the literature, in this paper we will derive some important implications for the firms' equilibrium strategies under the combined supply modes.

To this end, we construct a decentralized assembly system that contains two sources of uncertainty. On one hand, the market demand is stochastic so that the assembler and the supplier have to make their procurement/production decisions before the exact demand is realized. On the other hand, we assume that supplier l's production is perfectly reliable, while supplier 2 has a stochastic production yield rate. The assembler has to preorder the components from supplier 1 under the ordering mode but is also able to exert the VMI mode on supplier 2. Consequently, the assembler and supplier 2 have to simultaneously choose their production and procurement quantities in consideration of the other's response. This setting helps us to solve the following questions. First, what are the firms equilibrium production and procurement strategies in this decentralized assembly system with dual supply modes? Second, compared to the centralized system how to achieve the supply chain coordination? Third, what are the differences among different supply modes, for example, ordering mode versus VMI mode versus combined mode?

Given the equilibrium strategies, our analysis has the following observations. First, double marginalization significantly undermines the channel's efficiency, in which case both suppliers produce fewer components than that in the centralized system. In particular, the supplier with random yield produces more components than his partner's. Second, to improve the channel efficiency, we incorporate an advance payment contract. When the sharing parameter (donated as $\lambda$ ) falls into a rational range, the suppliers achieve perfect coordination. Moreover, we show that from the channel's perspective, ordering mode dominates both the combined mode and VMI mode. This result coincides with the supplier's interest but goes against the assembler's profit. The intuition is that for any firm, the better operational mode always mitigates its inventory risk. Overall, this paper speaks to the interactions between the assembler and the suppliers under dual supply modes in a decentralized system.

Besides the literature reviewed above, another stream of the related research is random yield and random demand. Note that this issue has been extensively studied by many scholars [6-10]. However, as aforementioned the majority of these models have been developed under the condition that all the suppliers generate either production yield uncertainty or demand uncertainty, while in this paper, we assume that one supplier is perfectly reliable while the other supplier has a random yield issue. This setting is prevalent in both literature $[11,12]$ and practice. For example, the key component providers in assembly system (e.g., Intel) are always stable in their production capacity and can operate quite well. Therefore, they are nearly perfectly reliable. In contrast, the other component providers may suffer yield uncertainty, because they are usually in small size and unreliable in managing the production. One notable paper is Pan and So [13], in which they analyze an assemble-to-order system with these two types of supply. In their paper, assembler determines both kinds of suppliers' production input quantity in the interest of maximizing system's profit when facing pricedependent demand. Different from them, in our research there are multiple decision makers: the assembler decides the production quantity, while the supplier determines his production quantity.

The remainder of this paper is organized as follows. In Section 2, we describe the model setting. The equilibrium decisions of both decentralized and centralized systems are derived in Section 3. Section 4 investigates supply chain coordination and numerical examples. Section 5 concludes the paper and discusses some future researches.

\section{Model Descriptions}

Consider a decentralized assembly system that consists of two suppliers and one assembler, in which all the participants are risk-neutral and in purpose of maximizing their own profits. The final product consists of two components, as we define them as the key component and the matching component. The key component is provided by supplier 1 under ordering mode, and its unit production cost is $c_{1}$ and the unit wholesale price is $w_{1}\left(w_{1} \geq c_{1}\right)$. Differently, supplier 2 produces the matching component under VMI mode with unit production $\operatorname{cost} c_{2}$, and the unit wholesale price for component 2 is $w_{2}\left(w_{2} \geq c_{2}\right)$. We assume that supplier l's production yield is perfectly reliable, while supplier 2 has a random production yield. In particular, if supplier 2's initial input quantity is $q$, finally the output quantity of the components (that meet the quality level) is $\theta q . \theta$ is a random variable that falls into $[0,1]$ with probability distribution function $g(\cdot)$ and cumulative distribution function $G(\cdot)$ [13-15]. On the other hand, the market demand $D$ is also stochastic and follows the probability distribution function $f(\cdot)$ and cumulative distribution function $F(\cdot)$ in $[0, \infty)$. Without loss of generality, we assume that the final product's unit price is $p\left(p \geq w_{1}+w_{2}\right)$ and the assembly cost equals 0 (actually, if the cost $c_{a}>0$, the final product's unit price can be modified to $p^{\prime}=p+c_{a}$ ).

In a single-period setting, facing a random market demand $D$, the assembler determines the order quantity $Q_{1}$ from supplier 1 and takes the corresponding cost of overstock and shortage. This is the interaction between supplier 1 and assembler under ordering mode. In contrast, under the VMI contract with supplier 2, supplier 2 is the only decision maker that determines the input quantity $Q_{2}$ of matching component and will not receive the payment until the component is consumed. After both components' production and delivery are finished, the assembler assembles the components together and sells them to the customer. Once the overall product quantity is lower than the market demand ( $\left.\min E\left(Q_{1}, \theta Q_{2}\right)<D\right)$, the assembler will be penalized by the customer for shortage. The unit penalty cost is $\beta$. At the same time, if supplier 2's production quantity $\theta Q_{2}$ is insufficient $\left(\theta Q_{2}<D\right)$, he will also receive the punishment from the 
assembler. The unit penalty cost is $\beta_{2}\left(\beta_{2} \leq \beta\right)$. Besides, we normalize the salvage value of mismatched components to zero.

In summary, in this assembly system the assembler and supplier 2 simultaneously decide the production input quantity of the key component and the matching component. Therefore, we can develop a static Nash game model between the assembler and supplier 2 to derive their equilibrium strategies. In the following section, we first set up a benchmark by investigating the centralized system. After that, we focus on the decentralized system with dual supply modes. As a matter of convenience, we use $X^{d}$ to denote the condition in the decentralized system and $X^{\mathcal{C}}$ to represent the condition in the centralized system.

\section{System's Optimal Decisions}

In this section, we first set up a benchmark by studying the centralized system. Afterwards, we study the Nash game between the assembler and supplier 2 in the decentralized system. Comparing the channel's performances under these two scenraios, we finally discuss the supply chain coordination mechanism in the system.

3.1. Centralized System. In a centralized system, the assembler and the two suppliers will cooperate as one to achieve the system's highest performance. Therefore, we first formulate a centralized system's (denoted as $B$ ) expected profit function as follows:

$$
\begin{aligned}
\Pi_{B}^{c}\left(Q_{1}, Q_{2}\right)= & p E\left[\min \left(Q_{1}, \theta Q_{2}, D\right)\right]-\left(c_{1} Q_{1}+c_{2} Q_{2}\right) \\
& -\beta E\left[D-\min \left(Q_{1}, \theta Q_{2}\right)\right]^{+}
\end{aligned}
$$

Note that if the channel is centralized, its payoff is entirely determined by the production quantities of two components. As in (1), the first term is the final product's expected sales revenue. The second term is the production cost of both the key and the matching components. The third term is the expected penalty when stock-out occurs. Fixing either $Q_{i}(i=1,2)$, the maximization of $\Pi_{B}^{c}\left(Q_{1}, Q_{2}\right)$ becomes a news-vendor problem. Thus, we can use the following lemma to characterize the property of $\Pi_{B}^{c}\left(Q_{1}, Q_{2}\right)$. Note that throughout paper, all the proofs of lemmas and theorems are given in the appendix.

Lemma 1. The centralized system's expected profit function, $\Pi_{B}^{c}\left(Q_{1}, Q_{2}\right)$, is jointly concave in $Q_{1} \in[0, \infty)$ and $Q_{2} \in[0, \infty)$. The unique optimal input quantity of both the key and the matching components, $Q_{1}^{c *}$ and $Q_{2}^{c *}$, meets the F.O.Cs:

$$
\begin{aligned}
& \frac{\partial \Pi_{B}^{c}\left(Q_{1}, Q_{2}\right)}{\partial Q_{2}} \\
& \quad=(p+\beta) \int_{0}^{Q_{1} / Q_{2}} \int_{\theta Q_{2}}^{\infty} \theta f(D) g(\theta) d D d \theta-c_{2}=0,
\end{aligned}
$$

$$
\begin{aligned}
& \frac{\partial \Pi_{B}^{c}\left(Q_{1}, Q_{2}\right)}{\partial Q_{1}} \\
& \quad=(p+\beta) \int_{Q_{1} / Q_{2}}^{1} \int_{Q_{1}}^{\infty} f(D) g(\theta) d D d \theta-c_{1}=0 .
\end{aligned}
$$

From Lemma 1, we can always find the unique pair of $\left(Q_{1}^{c *}, Q_{2}^{c *}\right)$ that maximizes $\Pi_{B}^{c}\left(Q_{1}, Q_{2}\right)$. Moreover, we show that (2) and (3) are symmetric and irrespective to the parameter $\theta$. Given so, we can derive the relationship of $Q_{1}^{x *}$ and $Q_{2}^{x *}$ ( $x$ represents $d$ and $c$ ). The following theorem states that $Q_{2}^{x *}>Q_{1}^{x *}$ always holds.

Theorem 2. In centralized system, the optimal input quantity of the matching component is strictly larger than the optimal input quantity of the key component, that is, $Q_{2}^{c *}>Q_{1}^{c *}$.

The intuition behind Theorem 2 can be explained as follows. Consider that with random yield, if the input quantity of matching component is fewer than that of the key component, the output $\theta Q_{2}$ must be eternally fewer than $Q_{1}$. This implies that the penalty cost of matching component's shortage can be reduced by enlarging its input quantity, which is also beneficial to supplier 2 and the entire system. Therefore, only if $Q_{2}$ exceeds $Q_{1}$, they can reach an equilibrium. Now, we have derived the equilibrium decisions in the centralized system. This will be compared with the following scenario wherein the two players make their decisions independently.

3.2. Decentralized System. In the decentralized system, assembler (denoted as $A$ ) will pay supplier 1 (denoted as $S_{1}$ ) immediately after he finishes the key component's delivery. Therefore, his profit function is $\Pi_{S_{1}}^{d}=\left(w_{1}-c_{1}\right) Q_{1}$, which is nonnegative and meets the participant constraint.

As to supplier 2 (denoted as $S_{2}$ ), he will not receive the payment until the matching component is consumed. Besides, supplier 2 will be penalized by the assembler if his output is less than the customer's demand. Thus, supplier 2's expected profit function can be formulated as

$$
\Pi_{S_{2}}^{d}=w_{2} E\left[\min \left(Q_{1}, \theta Q_{2}, D\right)\right]-c_{2} Q_{2}-\beta_{2} E\left[D-\theta Q_{2}\right]^{+} .
$$

In (4), the first term is the expected revenue. The second term is the production cost, which is based on the input quantity. The third term stands for the expected penalty when the stock out of the matching component occurs. Similarly, we have the assembler's expected profit function as follows:

$$
\begin{aligned}
\Pi_{A}^{d}= & \left(p-w_{2}\right) E\left[\min \left(Q_{1}, \theta Q_{2}, D\right)\right]-w_{1} Q_{1} \\
& -\beta E\left[D-\min \left(Q_{1}, \theta Q_{2}\right)\right]^{+}+\beta_{2} E\left[D-\theta Q_{2}\right]^{+} .
\end{aligned}
$$

Note that in the above equation, the first two terms are the final product's expected sales revenue minus the procurement cost of the key and the matching components. The third term is the penalty penalized by the customer when the stock-out occurs. The fourth one is the compensation from supplier 2 
when his yield cannot meet the customer's demand. The following lemma demonstrates that the objective functions, $\Pi_{S_{2}}^{d}$ and $\Pi_{A}^{d}$, are concave in supplier 2's and supplier 1's production (input) quantity, respectively.

Lemma 3. Let $Q_{i}^{d *}\left(Q_{j}\right)$ denote the optimal production input quantity of the key/matching component for a given $Q_{j}(i, j=$ $1,2 ; i \neq j)$. Then, we have the following.

(i) Supplier 2's profit function, $\Pi_{S_{2}}^{d}$, is concave in $Q_{2} \in$ $[0, \infty)$. And the optimal input quantity $Q_{2}^{d *}\left(Q_{1}\right)$ meets the first-order condition:

$$
\begin{aligned}
\frac{\partial \Pi_{S_{2}}^{d}\left(Q_{1}, Q_{2}\right)}{\partial Q_{2}}= & w_{2} \int_{0}^{Q_{1} / Q_{2}} \int_{\theta Q_{2}}^{\infty} \theta f(D) g(\theta) d D d \theta \\
& +\beta_{2} \int_{0}^{1} \int_{\theta Q_{2}}^{\infty} \theta f(D) g(\theta) d D d \theta-c_{2}=0
\end{aligned}
$$

(ii) The assembler's profit function, $\Pi_{A}^{d}$, is concave in $Q_{1} \in$ $[0, \infty)$. And, the optimal input quantity $Q_{1}^{d *}\left(Q_{2}\right)$ meets the first-order condition:

$$
\begin{aligned}
& \frac{\partial \Pi_{A}^{d}\left(Q_{1}, Q_{2}\right)}{\partial Q_{1}} \\
& \quad=\left(p+\beta-w_{2}\right) \int_{Q_{1} / Q_{2}}^{1} \int_{Q_{1}}^{\infty} f(D) g(\theta) d D d \theta-w_{1}=0 .
\end{aligned}
$$

With Lemma 3, we can characterize the optimal production quantity of both key and matching components through their first-order-conditions (F.O.Cs) and the static Nash game equilibrium. In particular, we have the following theorem.

Theorem 4. There is a unique static Nash equilibrium solution $D\left(Q_{1}^{d *}, Q_{2}^{d *}\right)$ to the assembler's and supplier 2's decisions of the key and the matching components' production quantity. The solution $\left(Q_{1}^{d *}, Q_{2}^{d *}\right)$ meets the combination of (6) and (7).

Theorem 4 derives the unique optimal production quantity of the key component $\left(Q_{1}^{d *}\right)$ and the matching component $\left(Q_{2}^{d *}\right)$, which are given under the exogenous operational parameters, for example, the wholesale price $w_{1}$ and $w_{2}$. These indicate the highest payoffs that the assembler and suppliers can achieve in the decentralized system, which are certainly lower than those in the centralized system. Therefore, to identify the gap (inefficiency) between these two scenarios (decentralized versus centralized), we have the following theorem.

Theorem 5. In the decentralized system, the summation of all the participants' optimal profit is strictly less than the centralized system's optimal total profit, that is,

$$
\left.\left(\Pi_{S_{1}}^{d}+\Pi_{S_{2}}^{d}+\Pi_{A}^{d}\right)\right|_{Q_{1}=Q_{1}^{d *}, Q_{2}=Q_{2}^{d *}}<\Pi_{B}^{c}\left(Q_{1}^{c *}, Q_{2}^{c *}\right) .
$$

From Theorem 5, we can see that although there exists a unique static Nash equilibrium in the decentralized system, it is necessary to introduce a proper contract to coordinate the supply chain to move like a centralized system. Therefore, we propose an advance payment contract to achieve the supply chain coordination.

3.3. Supply Chain Coordination. Among the previous literature, a number of different contract types aiming at coordinating the supply chain are discussed, for example, the revenue sharing contract, the buy-back contract, and so forth. For a detailed review, please refer to Cachon [16]. Differently, in this paper we focus on another contract type: advance payment contract. That is, at the beginning of a production period, the assembler pays $\left(\lambda_{1} \Pi_{B}^{c *}+c_{1} Q_{1}^{c *}\right)$ to supplier 1 and $\left(\lambda_{2} \Pi_{B}^{c *}+c_{2} Q_{2}^{c *}\right)$ to supplier 2 . After receiving the customer's order, the assembler announces the production input quantity of both the key and the matching components, $Q_{i}^{c *}(i=1,2)$. Afterwards, the suppliers carry out the production according to their announcement. Finally, the assembler assembles the components into the products and sells them to the customer. Accordingly, he obtains the profit of $\left(1-\sum_{i=1}^{2} \lambda_{i}\right) \Pi_{B}^{c *}$, where $\lambda_{i} \in[0,1]$ and $\sum_{i=1}^{2} \lambda_{i} \leq 1$.

Note that the major feature of the above contract is that the assembler should pay the suppliers in advance. Thus, if supplier $i$ 's profit $\left(\lambda_{i} \Pi_{B}^{c *}\right)$ is less than what he can gain in the decentralized system, the contract will fail in practice. Therefore, we have the following theorem to state that there always exists a proper pair of $\left(\lambda_{1}, \lambda_{2}\right)$ which can successfully implement the contract.

Theorem 6. The decentralized assembly system with dual supply modes always can be coordinated through the advance payment contract. In which, the contract parameter $\lambda_{i}$ meets the following conditions:

$$
\frac{\Pi_{S_{i}}^{d *}}{\Pi_{B}^{c *}} \leq \lambda_{i} \leq 1-\frac{\Pi_{A}^{d *}+\Pi_{S_{j}}^{d *}}{\Pi_{B}^{c *}}, \quad \sum_{i=1}^{2} \lambda_{i} \leq 1-\frac{\Pi_{A}^{d *}}{\Pi_{B}^{c *}},
$$

wherein $\Pi_{y}^{d *}=\Pi_{y}^{d}\left(Q_{1}^{d *}, Q_{2}^{d *}\right), y \in\left\{S_{1}, S_{2}, A\right\}$, and $i, j=1,2$, $i \neq j$.

With Theorem 6, we can draw the insight that the advance payment successfully makes the suppliers operate as the assembler's subsidiaries, so as to achieve the supply chain coordination. Besides, under this contract the assembler actually changes the business scenario with supplier 2 by deciding the input quantity of the matching component himself. This implies that when facing two kinds of supply, the VMI arrangement with the supplier who generates yield uncertainty is not beneficial for the entire supply chain. To this point, we further study the comparison of the decentralized and centralized systems and investigate the effectiveness of the advance payment contract in the following section.

\section{Numerical Analysis}

In this section, we conduct two numerical examples. In the first example, we make a comparison between components' 
Table 1: Performance with different penalty, price, and demand.

\begin{tabular}{|c|c|c|c|c|c|c|c|c|c|c|c|}
\hline \multirow{2}{*}{ Demand } & \multirow{2}{*}{$x$} & \multicolumn{2}{|c|}{$\left(Q_{1}^{x *}, Q_{2}^{x *}\right)$} & \multicolumn{2}{|c|}{$\Pi_{S_{1}}^{x^{*}}$} & \multicolumn{2}{|c|}{$\Pi_{S_{2}}^{x *}$} & \multicolumn{2}{|c|}{$\Pi_{A}^{x *}$} & \multicolumn{2}{|c|}{$\sum_{y} \Pi_{y}^{x^{*}}$} \\
\hline & & Case 1 & Case 2 & Case 1 & Case 2 & Case 1 & Case 2 & Case 1 & Case 2 & Case 1 & Case 2 \\
\hline \multirow{3}{*}{$N(40,1.5)$} & $d$ & $(39.5,56.1)$ & $(39.8,60.1)$ & 79.00 & 39.80 & 32.46 & 54.15 & 184.05 & 162.60 & 295.51 & 256.54 \\
\hline & $d^{\prime}$ & $(40.6,70.9)$ & $(40.5,64.0)$ & 81.20 & 40.50 & 70.90 & 96.00 & 175.54 & 128.07 & 327.64 & 264.57 \\
\hline & $c$ & $(41.3,82.0)$ & $(41.0,78.8)$ & \multicolumn{6}{|c|}{1} & 333.63 & 274.89 \\
\hline \multirow{3}{*}{$N(35,2)$} & $d$ & $(34.3,48.6)$ & $(34.8,52.9)$ & 68.60 & 34.80 & 24.44 & 42.23 & 147.40 & 134.52 & 240.44 & 211.55 \\
\hline & $d^{\prime}$ & $(35.8,62.8)$ & $(35.6,56.5)$ & 71.60 & 35.60 & 62.80 & 84.75 & 139.21 & 98.96 & 273.61 & 219.31 \\
\hline & $c$ & $(36.7,73.4)$ & $(36.5,70.1)$ & \multicolumn{6}{|c|}{ I } & 279.39 & 229.05 \\
\hline \multirow{3}{*}{$N(45,1)$} & $d$ & $(44.6,62.6)$ & $(44.9,68.4)$ & 89.20 & 44.90 & 40.10 & 63.72 & 213.29 & 191.80 & 342.59 & 300.42 \\
\hline & $d^{\prime}$ & $(45.4,79.4)$ & $(45.3,71.7)$ & 90.80 & 45.30 & 79.40 & 107.56 & 208.46 & 153.89 & 378.66 & 306.75 \\
\hline & $c$ & $(45.8,92.0)$ & $(45.7,88.2)$ & \multicolumn{6}{|c|}{ I } & 384.89 & 318.10 \\
\hline
\end{tabular}

Case 1: $c_{1}=4, w_{1}=6, c_{2}=3, w_{2}=8, \beta_{2}=4, p=25$, and $\beta=15$.

Case 2: $c_{1}=4, w_{1}=5, c_{2}=3, w_{2}=9, \beta_{2}=5, p=23$, and $\beta=13$.

equilibrium quantities and firms' expected payoffs under three alternative scenarios: the decentralized system with dual supply modes, the decentralized system with ordering mode $[1,17]$, and the centralized system. This helps us to identify the magnitude of decentralization in the assembly system with dual supply modes. Second, we examine the robustness of advance payment contract on the supply chain coordination by making the sensitivity analysis of sharing rate $\lambda_{i}(i=1,2)$.

4.1. Comparison of the Alternative Scenarios. In Section 3.3, we have shown that dual supply modes is never beneficial to the entire supply chain, which is caused by the conflict between two supply modes: ordering mode and VMI mode. To better distinguish the difference between these two mode, we next introduce another scenario that the assembly system only contains ordering mode (denoted as $d^{\prime}$ ), wherein both the key and the matching components' input quantity are selected by the assembler. Intuitively, under such a circumstance the supply chain's performance should be better than that with dual supply modes; however, it is still worse than the centralized system since the decentralization still exists.

With only ordering mode, the assembler independently decides both the input quantities of key component and matching component. Therefore, each party's expected profit functions change to

$$
\begin{aligned}
\Pi_{S_{1}}^{d^{\prime}}= & \left(w_{1}-c_{1}\right) Q_{1}, \quad \Pi_{S_{2}}^{d^{\prime}}=w_{2} E\left[\theta Q_{2}\right]-c_{2} Q_{2}, \\
\Pi_{A}^{d^{\prime}}= & p E\left[\min \left(Q_{1}, \theta Q_{2}, D\right)\right]-w_{1} Q_{1}-w_{2} E\left(\theta Q_{2}\right) \\
& -\beta E\left[D-\min \left(Q_{1}, \theta Q_{2}\right)\right]^{+} .
\end{aligned}
$$

Following the similar principle of Lemma 1, we can easily find that (11) is jointly concave in $Q_{1}$ and $Q_{2}$, and the optimal $\left(Q_{1}^{d^{\prime} *}, Q_{2}^{d^{\prime} *}\right)$ meets the following F.O.Cs:

$$
\begin{aligned}
\frac{\partial \Pi_{A}^{d^{\prime}}\left(Q_{1}, Q_{2}\right)}{\partial Q_{1}}= & (p+\beta) \int_{0}^{Q_{1} / Q_{2}} \int_{\theta Q_{2}}^{\infty} \theta f(D) g(\theta) d D d \theta \\
& -w_{2} \int_{0}^{1} \theta g(\theta) d \theta=0,
\end{aligned}
$$

$$
\begin{aligned}
& \frac{\partial \Pi_{A}^{d^{\prime}}\left(Q_{1}, Q_{2}\right)}{\partial Q_{1}} \\
& \quad=(p+\beta) \int_{Q_{1} / Q_{2}}^{1} \int_{Q_{1}}^{\infty} f(D) g(\theta) d D d \theta-w_{1}=0 .
\end{aligned}
$$

Due to the F.O.Cs and the profit functions' complexity, we now make the comparison of the three alternative scenarios via numerical approaches. In the following numerical example, we conduct several comparisons under different groups of parameters.

Example 1. Assume that the customer's demand follows the normal distribution and the production random variable of supplier 2 follows the uniform distribution. See Table 1.

Note that Table 1 represents the key and the matching components' optimal input quantity, the expected profits of two suppliers and the assembler, and the expected total profit of the supply chain for three alternative scenarios. As observed from the above table, we have $\sum_{y} \Pi_{y}^{d *}<\sum_{y} \Pi_{y}^{d^{\prime} *}<$ $\Pi_{B}^{c *}$, which demonstrates that the decentralized systems generate incoordination, and the decentralized system only with ordering mode performs better than the one with dual supply modes. Specifically, both the key and the matching components' optimal input quantity in the decentralized systems are less than those in the centralized system, that is, $Q_{1}^{d *}<Q_{1}^{d^{\prime} *}<Q_{1}^{c *}$ and $Q_{2}^{d *}<Q_{2}^{d^{\prime} *}<Q_{2}^{c *}$, which directly makes the performance of decentralized systems become worse than the centralized system. Besides, there exist $\Pi_{S_{1}}^{d^{\prime} *}>\Pi_{S_{1}}^{d^{\prime} *}, \Pi_{S_{2}}^{d^{\prime} *}>\Pi_{S_{2}}^{d^{\prime} *}$, and $\Pi_{S_{2}}^{d^{\prime} *}<\Pi_{S_{2}}^{d *}$. This implies that from the supplier's perspective, they prefer to providing components with the ordering mode. In contrast, under the decentralized situation, the assembler will certainly choose the VMI mode with the supplier who generates yield uncertainty, even though this option significantly undermines the channel's performance. 

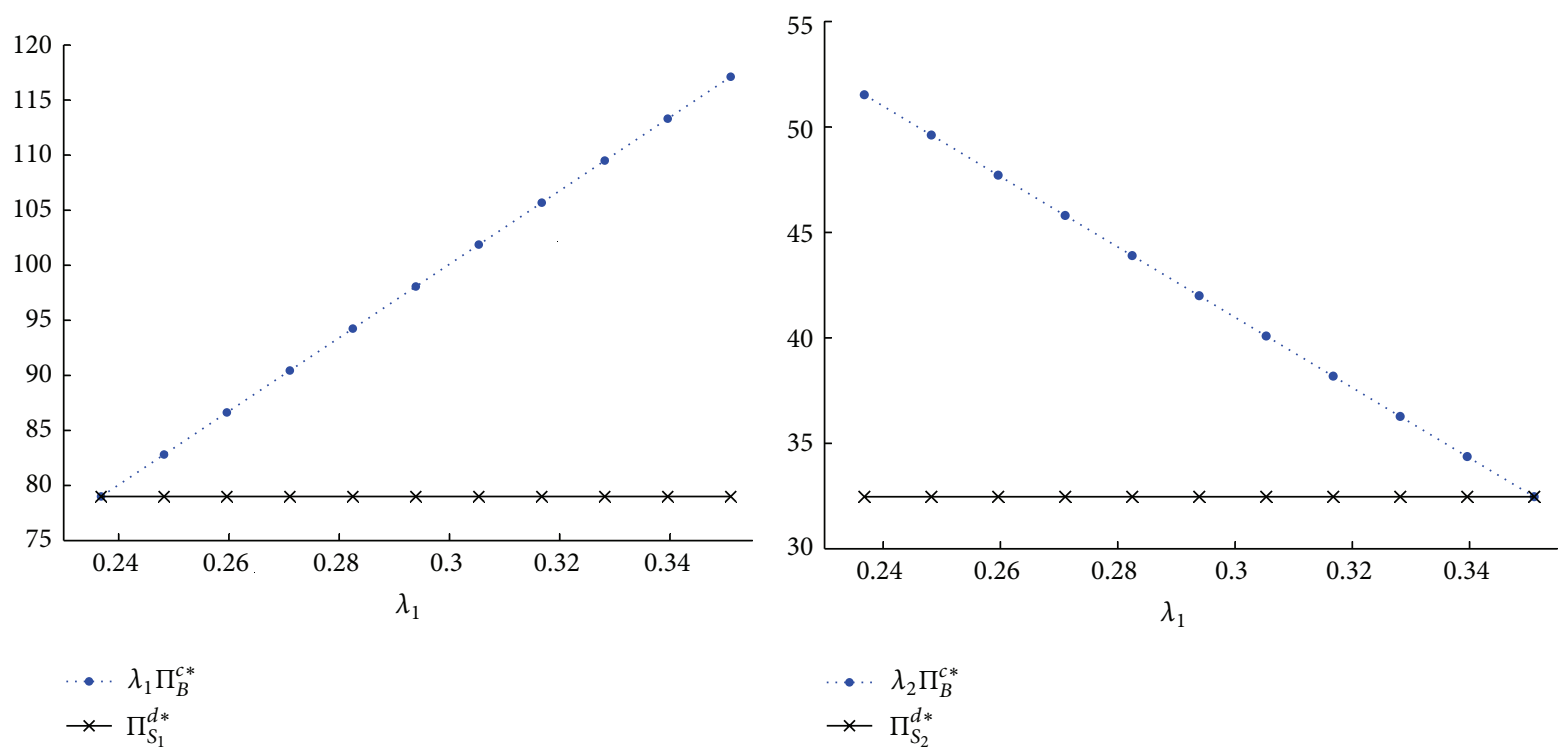

(a)

(b)

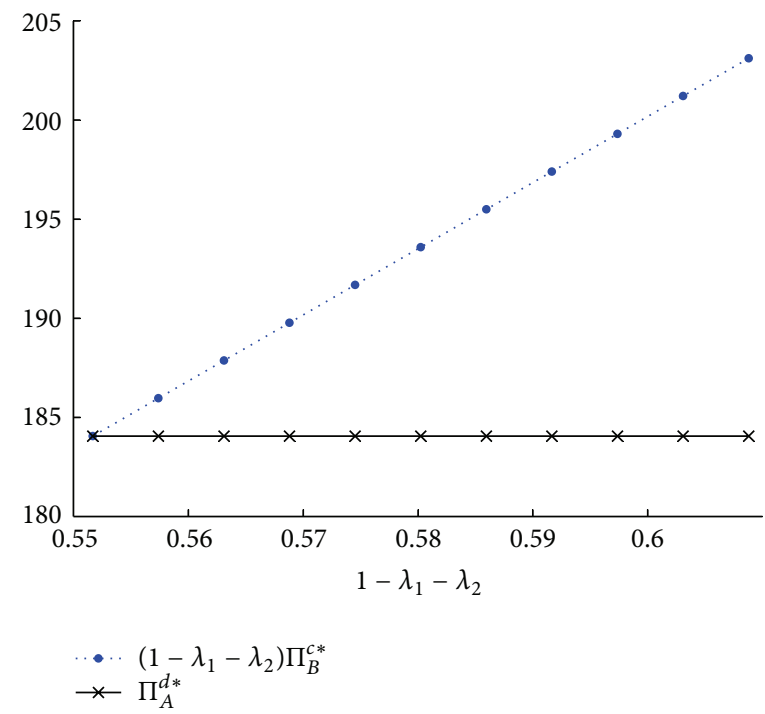

(c)

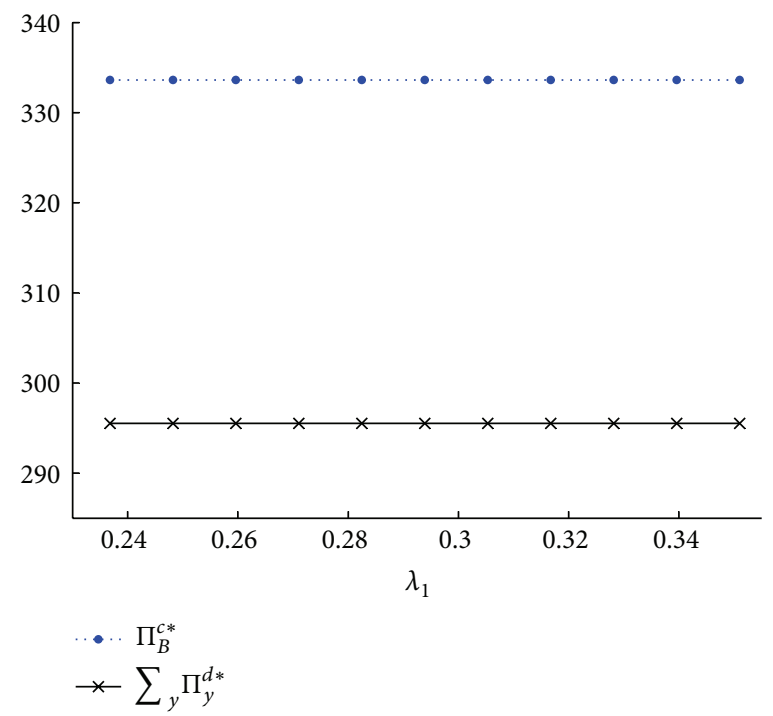

(d)

FIGURE 1: Effectiveness of the advance payment contract under different $\lambda_{i}$.

4.2. Sensitivity Analysis on $\lambda_{i}$. In the above discussion, we have shown that the advance payment contract can perfectly coordinate the decentralized supply chain by setting the appropriate $\lambda_{i}$. We next identify how the firms' equilibrium payoffs react to the variance of $\lambda_{i}$.

Example 2. Given the following data: $c_{1}=4, w_{1}=6, c_{2}=3$, $w_{2}=8, \beta_{2}=4, p=25, \beta=15 ; D \sim N(40,1.5)$, $\theta \sim U(0,1)$, let $\lambda_{1}$ take the value uniformly distributed over $\left[\left(\Pi_{S_{1}}^{d *} / \Pi_{B}^{c *}\right), 1-\left(\left(\Pi_{A}^{d *}+\Pi_{S_{2}}^{d *}\right) / \Pi_{B}^{c *}\right)\right]$ and $\lambda_{2}$ take the value of $\left[\left(1-\lambda_{1}-\left(\Pi_{A}^{d *} / \pi_{B}^{c *}\right)\right)+\left(\Pi_{S_{2}}^{d *} / \Pi_{B}^{c *}\right)\right] / 2$, which is in $\left[\left(\Pi_{S_{2}}^{d *} / \Pi_{B}^{c *}\right), 1-\left(\left(\Pi_{A}^{d *}+\Pi_{S_{1}}^{d *}\right) / \Pi_{B}^{c *}\right)\right]$. Figure 1 shows the effectiveness of the advance payment contract.
In Figure 1, we can see that as long as the $\lambda_{i}$ is in its valid interval, all the suppliers and the assembler can be better off and the decentralized system can be perfectly coordinated with the advance payment contract. Therefore, it is effective and practicable.

\section{Conclusion}

In this paper, we investigate the equilibrium production and procurement strategies in a decentralized assembly system consisting of a single assembler and two suppliers. In particular, the assembler orders the key component from supplier 1 who is perfectly reliable, while supplier 2 provides the matching component under VMI mode with yield uncertainty. We derive the components' optimal production input quantity in 
a static Nash game model and also set up a benchmark case by identifying the centralized system. Given the comparison between these two scenarios, we propose an advance payment contract to achieve supply chain coordination. We also make two numerical examples and find that (1) the less components' optimal production input quantity makes the decentralized systems perform worse than the centralized system, (2) the assembler prefers VMI mode in practice so as to maximize his own expected profit, and (3) advance payment contract is effective regardless the sharing rate.

One extension of our model is to consider a more general assembly system with one assembler and $N(\geq 3)$ suppliers, where the decisions of those suppliers with yield uncertainty can affect each other. Besides, it can be extended to other random yield models. For example, in semiconductor industry, the output of chips $Y(q)$ is a nonlinear function of the input of silicon wafers $q$, and other related factors. These extensions certainly have the potential to be better explored in the future.

\section{Appendix}

Proof of Lemma 1. Equation (1) can be rewritten as $\Pi_{B}^{c}\left(Q_{1}, Q_{2}\right)=p C-\beta D-\left(c_{1} Q_{1}+c_{2} Q_{2}\right)$, wherein

$$
\begin{aligned}
C= & \int_{Q_{1} / Q_{2}}^{1} \int_{Q_{1}}^{\infty} Q_{1} f(D) g(\theta) d D d \theta \\
& +\int_{0}^{Q_{1} / Q_{2}} \int_{\theta Q_{2}}^{\infty} \theta Q_{2} f(D) g(\theta) d D d \theta \\
& +\int_{0}^{Q_{1}} \int_{D / Q_{2}}^{1} D g(\theta) f(D) d \theta d D, \\
D= & \int_{Q_{1} / Q_{2}}^{1} \int_{Q_{1}}^{\infty}\left(D-Q_{1}\right) f(D) g(\theta) d D d \theta \\
& +\int_{0}^{Q_{1} / Q_{2}} \int_{\theta Q_{2}}^{\infty}\left(D-\theta Q_{2}\right) f(D) g(\theta) d D d \theta .
\end{aligned}
$$

Taking first- and second-order derivatives with respect to $Q_{1}$ and $Q_{2}$ in (1) separately, we get

$$
\begin{aligned}
\frac{\partial \Pi_{B}^{c}\left(Q_{1}, Q_{2}\right)}{\partial Q_{2}} & =(p+\beta) \int_{0}^{Q_{1} / Q_{2}} \int_{\theta Q_{2}}^{\infty} \theta f(D) g(\theta) d D d \theta-c_{2}, \\
\frac{\partial \Pi_{B}^{c}\left(Q_{1}, Q_{2}\right)}{\partial Q_{1}} & =(p+\beta) \int_{Q_{1} / Q_{2}}^{1} \int_{Q_{1}}^{\infty} f(D) g(\theta) d D d \theta-c_{1}, \\
\frac{\partial^{2} \Pi_{B}^{c}\left(Q_{1}, Q_{2}\right)}{\partial Q_{2}^{2}} & =-(p+\beta)\left(E+\frac{Q_{1}^{2}}{Q_{2}^{3}} F\right), \\
\frac{\partial^{2} \Pi_{B}^{c}\left(Q_{1}, Q_{2}\right)}{\partial Q_{1}^{2}} & =-(p+\beta)\left(G+\frac{1}{Q_{2}} F\right), \\
\frac{\partial^{2} \Pi_{B}^{c}\left(Q_{1}, Q_{2}\right)}{\partial Q_{1} \partial Q_{2}} & =(p+\beta) \frac{Q_{1}}{Q_{2}^{2}} F .
\end{aligned}
$$

Here, $E=\int_{0}^{\mathrm{Q}_{1} / \mathrm{Q}_{2}} \theta^{2} f\left(\theta \mathrm{Q}_{2}\right) g(\theta) d \theta>0, F=$ $\int_{\mathrm{Q}_{1}}^{\infty} f(D) g\left(Q_{1} / Q_{2}\right) d D>0$, and $G=\int_{Q_{1} / Q_{2}}^{1} f\left(Q_{1}\right) g(\theta) d \theta>0$. Then, we derive the Hessen Matrix as follows:

$$
\begin{aligned}
H & =\left|\begin{array}{cc}
\frac{\partial^{2} \Pi_{B}^{c}\left(Q_{1}, Q_{2}\right)}{\partial Q_{1}^{2}} & \frac{\partial^{2} \Pi_{B}^{c}\left(Q_{1}, Q_{2}\right)}{\partial Q_{1} \partial Q_{2}} \\
\frac{\partial^{2} \Pi_{B}^{c}\left(Q_{1}, Q_{2}\right)}{\partial Q_{2}^{2}} & \frac{\partial^{2} \Pi_{B}^{c}\left(Q_{1}, Q_{2}\right)}{\partial Q_{2} \partial Q_{1}}
\end{array}\right| \\
& =(p+\beta)\left|\begin{array}{cc}
-\left(G+\frac{1}{Q_{2}} F\right) & \frac{Q_{1}}{Q_{2}^{2}} F \\
\frac{Q_{1}}{Q_{2}^{2}} F & -\left(E+\frac{Q_{1}^{2}}{Q_{2}^{3}} F\right)
\end{array}\right| .
\end{aligned}
$$

In (A.3), the value of the first-order determinant is $-(p+$ $\beta)\left(G+\left(1 / Q_{2}\right) F\right)<0$ and the value of the second-order determinant is $(p+\beta)\left(E G+\left(Q_{1}^{2} / Q_{2}^{3}\right) F G+\left(1 / Q_{2}\right) E F\right)>0$. As such, the Hessen Matrix is negative definite. Therefore, $\Pi_{B}^{c}\left(Q_{1}, Q_{2}\right)$ is joint concave in $Q_{1} \in[0, \infty)$ and $Q_{2} \in[0, \infty)$. And the optimal production input quantity $\left(Q_{1}^{c *}, Q_{2}^{c *}\right)$ meets the F.O.Cs, which consists of $\left(\partial \Pi_{B}^{c}\left(Q_{1}, Q_{2}\right)\right) / \partial Q_{2}=0$ and $\left(\partial \Pi_{B}^{c}\left(Q_{1}, Q_{2}\right)\right) / \partial Q_{1}=0$.

Proof of Lemma 3. Equation (4) can be rewritten as $\Pi_{S_{2}}^{d}=$ $w_{2} A-\beta_{2} B-c_{2} Q_{2}$, wherein

$$
\begin{aligned}
A= & \int_{\mathrm{Q}_{1} / \mathrm{Q}_{2}}^{1} \int_{\mathrm{Q}_{1}}^{\infty} Q_{1} f(D) g(\theta) d D d \theta \\
& +\int_{0}^{\mathrm{Q}_{1} / \mathrm{Q}_{2}} \int_{\theta \mathrm{Q}_{2}}^{\infty} \theta \mathrm{Q}_{2} f(D) g(\theta) d D d \theta \\
& +\int_{0}^{\mathrm{Q}_{1}} \int_{D / \mathrm{Q}_{2}}^{1} D g(\theta) f(D) d \theta d D, \\
B= & \int_{0}^{1} \int_{\theta \mathrm{Q}_{2}}^{\infty}\left(D-\theta \mathrm{Q}_{2}\right) f(D) g(\theta) d D d \theta .
\end{aligned}
$$

Fix $Q_{1}$, taking first- and second-order derivatives with respect to $Q_{2}$ in (4), we get

$$
\begin{aligned}
\frac{\partial \Pi_{S_{2}}^{d}}{\partial Q_{2}}= & w_{2} \int_{0}^{Q_{1} / Q_{2}} \int_{\theta Q_{2}}^{\infty} \theta f(D) g(\theta) d D d \theta \\
& +\beta_{2} \int_{0}^{1} \int_{\theta Q_{2}}^{\infty} \theta f(D) g(\theta) d D d \theta-c_{2} \\
\frac{\partial^{2} \Pi_{S_{2}}^{d}}{\partial Q_{2}^{2}}=- & -w_{2}\left\{\int_{0}^{Q_{1} / Q_{2}} \theta^{2} f\left(\theta Q_{2}\right) g(\theta) d \theta\right. \\
& \left.+\int_{Q_{1}}^{\infty} \frac{Q_{1}^{2}}{Q_{2}^{3}} f(D) g\left(\frac{Q_{1}}{Q_{2}}\right) d D\right\} \\
& -\beta_{2} \int_{0}^{1} \theta^{2} f\left(\theta Q_{2}\right) g(\theta) d \theta
\end{aligned}
$$


It is easy to find that $\partial^{2} \Pi_{S_{2}}^{d} / \partial Q_{2}^{2}<0$. Therefore, $\Pi_{S_{2}}^{d}$ is concave in $Q_{2}$. Besides, we observe that $\partial \Pi_{S_{2}}^{d} /\left.\partial Q_{2}\right|_{Q_{2}=0}=w_{2}+$ $\beta_{2} \bar{\theta}-c_{2}>0$ and $\partial \Pi_{S_{2}}^{d} /\left.\partial Q_{2}\right|_{Q_{2} \rightarrow \infty}=-c_{2}<0$. As such, there must exist a $Q_{2} \in[0, \infty)$ that meets the first-order condition $\partial \Pi_{S_{2}}^{d} / \partial Q_{2}=0$.

Similarly, fix $Q_{2}$, taking first- and second-order derivatives with respect to $Q_{1}$ in (5), we get

$$
\begin{aligned}
\frac{\partial \Pi_{A}^{d}}{\partial Q_{1}}=\left(p+\beta-w_{2}\right) \int_{Q_{1} / Q_{2}}^{1} \int_{Q_{1}}^{\infty} f(D) g(\theta) d D d \theta-w_{1}, \\
\frac{\partial^{2} \Pi_{A}^{d}}{\partial Q_{1}^{2}}=-\left(p+\beta-w_{2}\right) \\
\times\left\{\int_{Q_{1} / Q_{2}}^{1} f\left(Q_{1}\right) g(\theta) d \theta\right. \\
\left.+\frac{1}{Q_{2}} \int_{Q_{1}}^{\infty} f(D) g\left(\frac{Q_{1}}{Q_{2}}\right) d D\right\} .
\end{aligned}
$$

We can easily derive $\partial^{2} \Pi_{A}^{d} / \partial Q_{1}^{2}<0, \partial \Pi_{A}^{d} /\left.\partial Q_{1}\right|_{Q_{1}=0}=$ $p+\beta-w_{2}-w_{1}>0$, and $\partial \Pi_{A}^{d} /\left.\partial Q_{1}\right|_{Q_{1} \rightarrow \infty}=-w_{1}<0$. Therefore, we can claim that $\Pi_{A}^{d}$ is concave in $Q_{1}$ and the optimal $Q_{1}^{d *}\left(Q_{2}\right)$ meets the first-order condition $\partial \Pi_{A}^{d} / \partial Q_{1}=$ 0 .

Proof of Theorem 2. Simplifying (7) and (3), we haveing

$$
\begin{gathered}
{\left[1-F\left(Q_{1}^{d *}\right)\right] \times\left[1-G\left(\frac{Q_{1}^{d *}}{Q_{2}^{d *}}\right)\right]=\frac{w_{1}}{p+\beta-w_{2}},} \\
{\left[1-F\left(Q_{1}^{c *}\right)\right] \times\left[1-G\left(\frac{Q_{1}^{c *}}{Q_{2}^{c *}}\right)\right]=\frac{c_{1}}{p+\beta} .}
\end{gathered}
$$

Consider the value of $G\left(Q_{1}^{x *} / Q_{2}^{x *}\right)$. If $Q_{1}^{d *} \geq Q_{2}^{d *}$ or $Q_{1}^{c *} \geq Q_{2}^{c *}, G\left(Q_{1}^{x *} / Q_{2}^{x *}\right)=1$. As such, the left parts of (A.7) and (A.8) both equal 0. Meanwhile, the right part of (A.7) and (A.8) both are larger than 0. Thus, both (A.7) and (A.8) cannot be balanced unless $Q_{1}^{x *}<Q_{2}^{x *}$.

Proof of Theorem 4. Considering the profit curves of supplier 2 and the assembler in (4) and (5), we let

$$
\begin{aligned}
& N_{1}\left(Q_{1}, Q_{2}\right)=\frac{\partial \Pi_{A}^{d}\left(Q_{1}, Q_{2}\right)}{\partial Q_{1}}, \\
& N_{2}\left(Q_{1}, Q_{2}\right)=\frac{\partial \Pi_{S_{2}}^{d}\left(Q_{1}, Q_{2}\right)}{\partial Q_{2}} .
\end{aligned}
$$

Taking first-order derivative with respect to $Q_{1}$ in $N_{1}\left(Q_{1}, Q_{2}\right)$ and $N_{2}\left(Q_{1}, Q_{2}\right)$, we have

$$
\begin{aligned}
& \frac{\partial N_{1}\left(Q_{1}, Q_{2}\right)}{\partial Q_{1}} \\
& =\frac{\partial^{2} \Pi_{A}^{d}\left(Q_{1}, Q_{2}\right)}{\partial Q_{1}^{2}} \\
& =-\left(p+\beta-w_{2}\right)\left\{\int_{Q_{1} / Q_{2}}^{1} f\left(Q_{1}\right) g(\theta) d \theta\right. \\
& \left.\quad+\frac{1}{Q_{2}} \int_{Q_{1}}^{\infty} f(D) g\left(\frac{Q_{1}}{Q_{2}}\right) d D\right\}<0, \\
& \frac{\partial N_{2}\left(Q_{1}, Q_{2}\right)}{\partial Q_{1}}=\frac{\partial^{2} \Pi_{S_{2}}^{d}\left(Q_{1}, Q_{2}\right)}{\partial Q_{2} \partial Q_{1}}=w_{2} \int_{Q_{1}}^{\infty} \frac{Q_{1}}{Q_{2}^{2}} f(D) g\left(\frac{Q_{1}}{Q_{2}}\right) d D>0 .
\end{aligned}
$$

Then, we get $\left(\partial N_{1}\left(Q_{1}, Q_{2}\right) / Q_{1}\right)-\left(\partial N_{2}\left(Q_{1}, Q_{2}\right) / Q_{1}\right)<$ 0 , for all $\left(Q_{1}, Q_{2}\right)$. As such, the players profit functions, $\Pi_{S_{2}}\left(Q_{1}, Q_{2}\right)$ and $\Pi_{A}\left(Q_{1}, Q_{2}\right)$, will meet once at most [3, Proposition 2.1]. Also, referring to Theorem 2.4 in Friedman [18], there is always a Nash equilibrium existing for concavepayoff-functions game like ours. Therefore, the static Nash equilibrium of both component's production input quantity is unique and the solution, $\left(Q_{1}^{d *}, Q_{2}^{d *}\right)$, meets the combination of (6) and (7).

Proof of Theorem 5. Sum $\Pi_{S_{1}}^{d}$, (4), and (5), we get the decentralized system's total profit function:

$$
\begin{aligned}
\Pi_{S_{1}}^{d}+\Pi_{S_{2}}^{d}+\Pi_{A}^{d}= & p E\left\{\min \left(Q_{1}, \theta Q_{2}, D\right)\right\}-\left(c_{1} Q_{1}+c_{2} Q_{2}\right) \\
& -\beta E\left[D-\min \left(Q_{1}, \theta Q_{2}\right)\right]^{+} .
\end{aligned}
$$

Notice that the formulation is the same as the one of the centralized system. As such, we can compare their optimal performance through the components' optimal production input quantity. Considering (A.7) and (A.8), we have

$$
\frac{w_{1}}{p+\beta-w_{2}}-\frac{c_{1}}{p+\beta}=\frac{(p+\beta)\left(w_{1}-c_{1}\right)+c_{1} w_{2}}{(p+\beta)\left(p+\beta-w_{2}\right)}>0 .
$$

As a result, we can derive that $\left(Q_{1}^{d *}, Q_{2}^{d *}\right) \neq\left(Q_{1}^{c *}, Q_{2}^{c *}\right)$. Otherwise, if $Q_{1}^{d *}=Q_{1}^{c *}$ and $Q_{2}^{d *}=Q_{2}^{c *}$, (A.7) and (A.8) cannot hold simultaneously. Then, plug $\left(Q_{1}^{d *}, Q_{2}^{d *}\right)$ and $\left(Q_{1}^{c *}, Q_{2}^{c *}\right)$ into the system's profit function separately; we can easily get

$$
\left.\left(\Pi_{S_{1}}^{d}+\Pi_{S_{2}}^{d}+\Pi_{A}^{d}\right)\right|_{Q_{1}=Q_{1}^{d *}, Q_{2}=Q_{2}^{d *}}<\Pi_{B}^{c}\left(Q_{1}^{c *}, Q_{2}^{c *}\right),
$$

since $\left(Q_{1}^{c *}, Q_{2}^{c *}\right)$ is the unique optimal decision. 
Proof of Theorem 6. Under the advance payment contract, each party's expected profit (denoted as $\Pi_{y}^{\mathrm{co}}$ ) can be formulated as follows:

$$
\Pi_{S_{1}}^{\mathrm{co}}=\lambda_{1} \Pi_{B}^{c *}, \quad \Pi_{S_{2}}^{\mathrm{co}}=\lambda_{2} \Pi_{B}^{c *}, \quad \Pi_{A}^{\mathrm{co}}=\left(1-\sum_{i=1}^{2} \lambda_{i}\right) \Pi_{B}^{c *} .
$$

To ensure the contract's success, one must satisfy the following constraints:

$$
\Pi_{y}^{\mathrm{co}} \geq \Pi_{y}^{d *}, \quad \forall y \in\left\{S_{1}, S_{2}, A\right\} .
$$

Substituting $\lambda_{i}(i=1,2)$ into (A.15) and combining with $\Pi_{B}^{c *}>\sum_{x} \Pi_{x}^{d *}$, we can easily derive that $\lambda_{i}$ meets equation (9).

\section{Acknowledgment}

This work was supported by the National Natural Science Foundation of China (nos. 71231007, 71102174, and 71372019).

\section{References}

[1] C. A. Yano, "Stochastic lead times in two-level assembly systems," IIE Transactions, vol. 19, no. 4, pp. 371-378, 1987.

[2] O. Tang and R. W. Grubbstrom, "The detailed coordination problem in a two-level assembly system with stochastic lead times," International Journal of Production Economics, vol. 8182, pp. 415-429, 2003.

[3] H. Gurnani and Y. Gerchak, "Coordination in decentralized assembly systems with uncertain component yields," European Journal of Operational Research, vol. 176, no. 3, pp. 1559-1576, 2007.

[4] D. Granot and S. Yin, "Competition and cooperation in decentralized push and pull assembly systems," Management Science, vol. 54, no. 4, pp. 733-747, 2008.

[5] Y. Gerchak and Y. Wang, "Revenue-sharing vs. wholesaleprice contracts in assembly systems with random demand," Production and Operations Management, vol. 13, no. 1, pp. 23-33, 2004.

[6] Y. Gerchak, Y. Wang, and C. A. Yano, "Lot sizing in assembly systems with random component yields," IIE Transactions, vol. 26, no. 2, pp. 19-24, 1994.

[7] H. Gurnani, R. Akella, and J. Lehoczky, "Supply chain management in assembly systems with random yield and random demand," IIE Transactions, vol. 32, no. 8, pp. 701-714, 2000.

[8] M. G. Güler and T. Bilgiç, "On coordinating an assembly system under random yield and random demand," European Journal of Operational Research, vol. 196, no. 1, pp. 342-350, 2009.

[9] Y. B. Xiao, J. Chen, and C. Y. Lee, "Optimal decisions for assemble-to-order systems with uncertain assembly capacity," International Journal of Production Economics, vol. 123, no. 1, pp. 155-165, 2010.

[10] J. Feng, L. Liu, and Y.-W. Wan, "Irreducibility of joint inventory positions in an assemble-to-order system under $(r, n q)$ policies," Naval Research Logistics, vol. 59, no. 1, pp. 18-25, 2012.

[11] S. Chopra, G. Reinhardt, and U. Mohan, "The importance of decoupling recurrent and disruption risks in a supply chain," Naval Research Logistics, vol. 54, no. 5, pp. 544-555, 2007.
[12] B. C. Giri, "Managing inventory with two suppliers under yield uncertainty and risk aversion," International Journal of Production Economics, vol. 133, no. 1, pp. 80-85, 2010.

[13] W. Pan and K. C. So, "Optimal product pricing and component production quantities for an assembly system under supply uncertainty," Operations Research, vol. 58, no. 6, pp. 1792-1797, 2010.

[14] C. A. Yano and H. L. Lee, "Lot sizing with random yields: a review," Operations Research, vol. 43, no. 2, pp. 311-334, 1995.

[15] H. Xu, "Managing production and procurement through option contracts in supply chains with random yield," International Journal of Production Economics, vol. 126, no. 2, pp. 306-313, 2010.

[16] G. P. Cachon, "Supply chain coordination with contracts," Handbooks in Operations Research and Management Science, no. 11, pp. 229-340, 2003.

[17] J. S. Song, C. A. Yano, and P. Lerssrisuriya, "Contract assembly: dealing with combined supply lead time and demand quantity uncertainty," Manufacturing \& Service Operations Management, vol. 2, no. 3, pp. 287-296, 2000.

[18] J. W. Friedman, Game Theory with Applications to Economics, Oxford University Press, New York, NY, USA, 1986. 


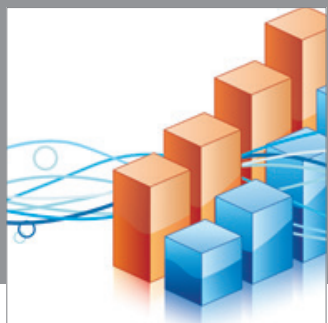

Advances in

Operations Research

mansans

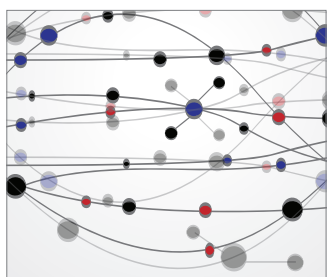

The Scientific World Journal
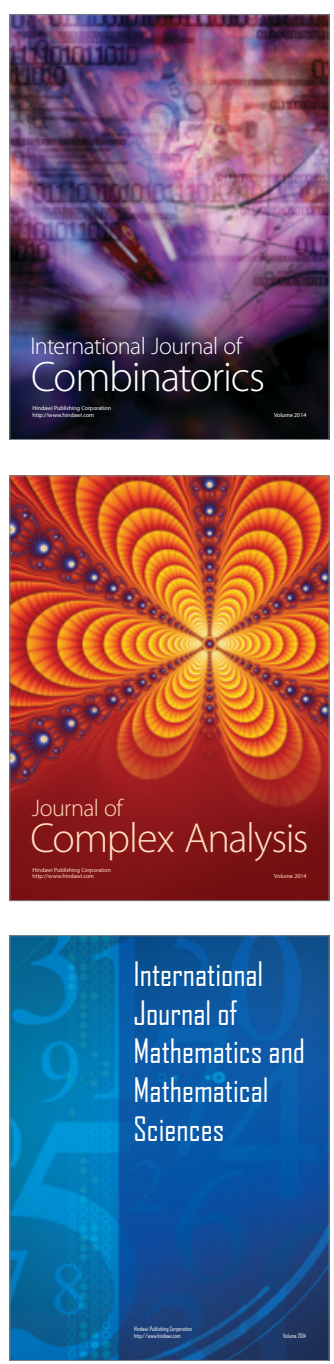
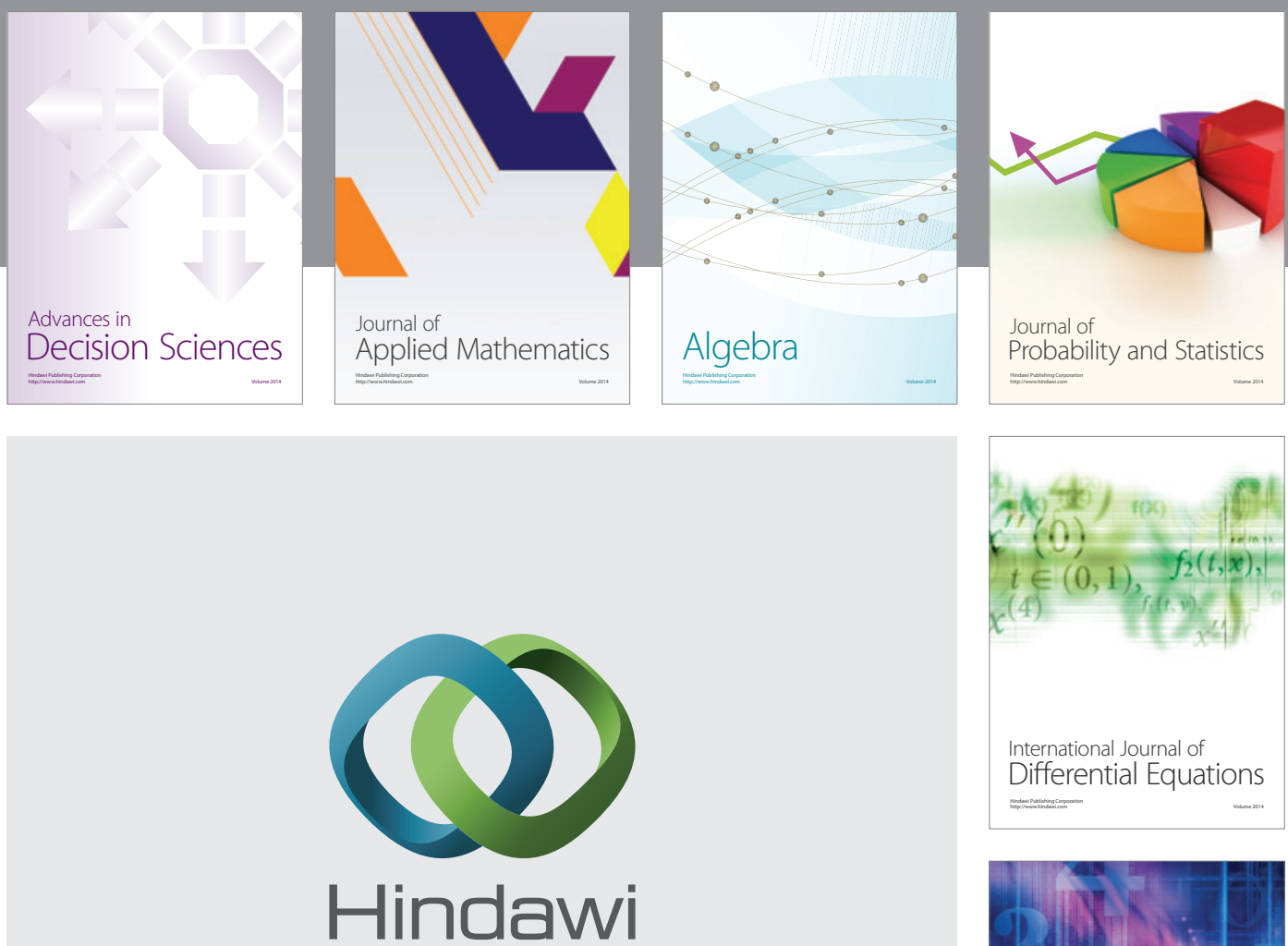

Submit your manuscripts at http://www.hindawi.com
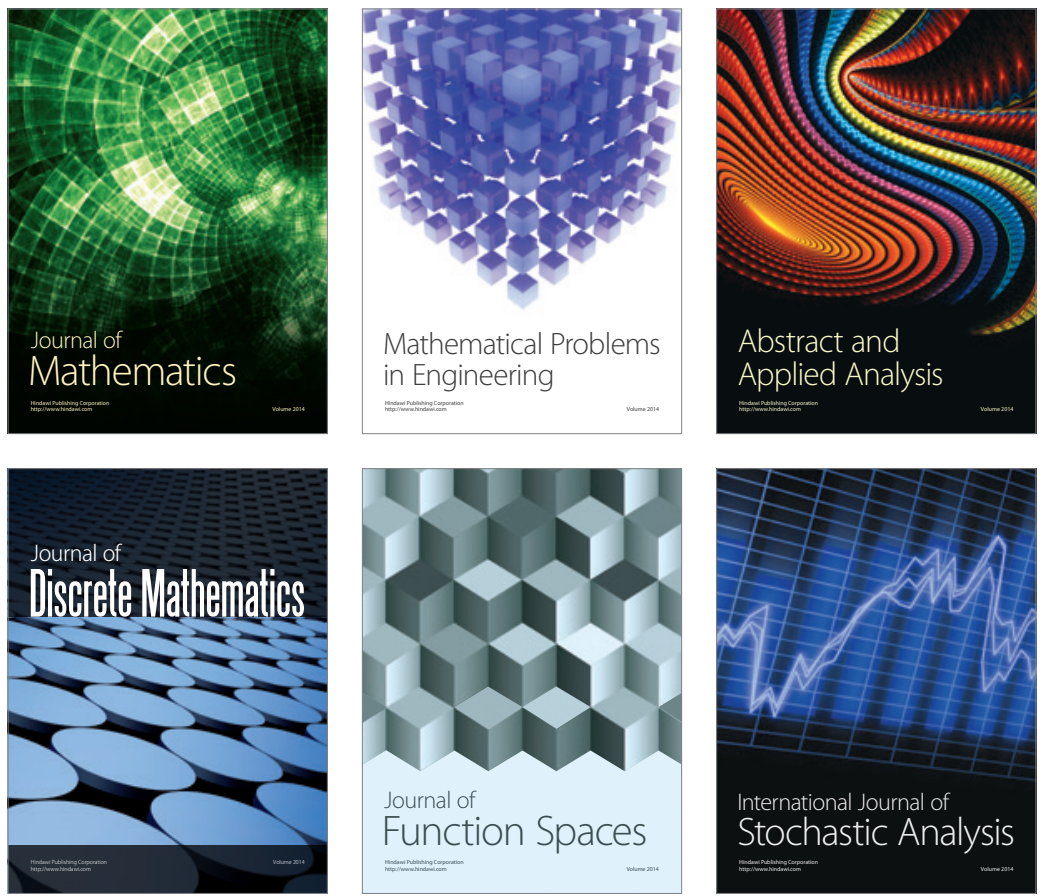

Journal of

Function Spaces

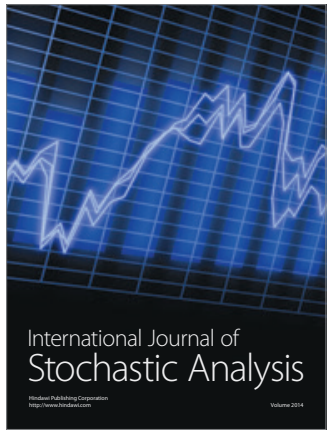

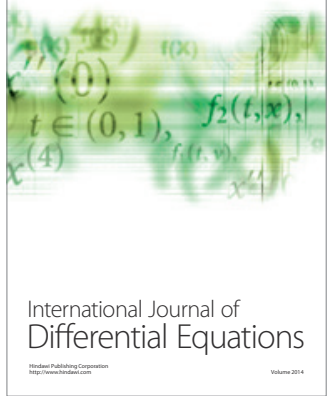
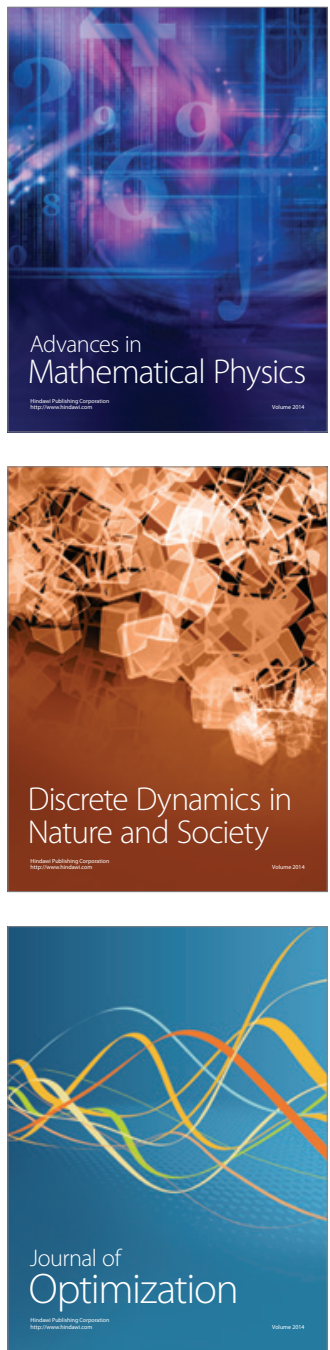